\title{
Laminar-turbulent transition regimes in the conical Taylor- Couette flow system
}

\author{
Fatma Yahi ${ }^{1,2,}{ }^{,}$, Ahcene Bouabdallah ${ }^{1}$, Yasmina Hamnoune ${ }^{1}$, Valéry Botton ${ }^{3}$, Daniel Henry ${ }^{3}$, Séverine Millet ${ }^{3}$, François \\ Rousset $^{4}$ and Takahiro Adachi ${ }^{5}$ \\ ${ }^{1}$ Thermodynamics and Energetic Systems Laboratory, Faculty of Physics, University of Sciences and Technology Houari Boumediene, \\ B.P. 32 El Alia 16111 Bab Ezzouar, Algiers, Algeria. \\ ${ }^{2}$ Genie Physical of Hydrocarbons Laboratory, Faculty of hydrocarbons and chemistry, University M'Hamed Bougara, 35000 \\ Boumerdes, Algeria. \\ ${ }^{3}$ Laboratoire de Mécanique des Fluides et d'Acoustique, CNRS/ Université de Lyon, Ecole Centrale de Lyon/Université Lyon 1/INSA de \\ Lyon, ECL, 36 Avenue Guy de Collongue, 69134 Ecully Cedex, France. \\ ${ }^{4}$ CETHIL, UMR CNRS 5008, Université de Lyon, INSA de Lyon/Université Lyon 1, INSA, Bâtiment Sadi Carnot, 9 rue de la Physique, \\ 69621 Villeurbanne Cedex, France. \\ ${ }^{5}$ Department of Mechanical Engineering, Akita University, Tegata-Gakuen 1-1, Akita 010-8502, Japan.
}

\begin{abstract}
The present work is intended to experimentally study the Taylor-Couette flow between coaxial cones. The inner cone is rotated and the outer cone is maintained fixed. Both cones have the same apex angle $\Phi=12^{\circ}$, giving a constant annular gap $\delta=0.12$. The height of the fluid column is $H=155 \mathrm{~mm}$. The working fluid is assumed as Newtonian and has constant properties (as density and viscosity) within the range of the required experimental conditions. By means of visualization techniques, the critical thresholds related to the onset of various instabilities have been obtained and the corresponding flow modes have been identified. Using images processing, spatio-temporal diagrams have also been calculated, showing the characteristics (wavelength, drift velocity) of the downward helical motion. The results obtained for these transition regimes are compared to those of Wimmer et al. [1-3].
\end{abstract}

\section{Introduction}

The study of instability phenomena in rotating systems remains very crucial for many industrial applications, as those concerned by mixing. Indeed, a better control of the processes could be obtained by a better understanding of the flow mechanisms involved and the way they vary with the governing parameters and the geometrical factors.

The investigation of the hydrodynamic instabilities in such systems has both objectives of predicting the critical parameters responsible for their onset and characterizing the flow which are thus triggered.

This kind of study in rotating systems can be helpful for the design of rotating machinery such as multiple concentric drives or turbine rotor, but also for the optimization of chemical equipment, such as compact rotating heat exchangers and mixers.

The viscous flow between concentric rotating cylinders has been widely studied. Concerning the conical geometry, Wimmer [1, 2, and 3] intensively investigated the Taylor-Couette flow in such geometry, showing experimentally that the flow is sensitive to the initial and boundary conditions, the gap size, the rotation velocity and the apex angle. Wimmer [1] demonstrated the coexistence of steady and unsteady flow regimes, which is not observed in the cylindrical geometry. Noui-
Mehidi et al. [4, 5, 6, 7] found, numerically, that when the apex angle $\Phi$ tends to $0^{\circ}$, the flow structure is identical to the classical Taylor-Couette flow and when the apex angle is increased towards $\Phi=180^{\circ}$, the vortex pair structure starts to break down and thereafter disappears. It is also observed that the flow develops similar characteristics as the flow between disks for an apex angle $\Phi>60^{\circ}[5]$.

Noui-Mehidi et al. [8] examined experimentally the acceleration effect of the inner cone rotation on the transition properties in a conical system, characterized by an apex angle equal to $\Phi=16.38^{\circ}$. They noted that the acceleration rate defined as $\mathrm{dRe} / \mathrm{dt}$ has a significant effect on the final flow: if the acceleration rate is less than 6.08 , a downward helical motion was observed, while for accelerations greater than 6.08, an upward helical motion took place. The acceleration rate can also affect the vortices number. It is also observed that, for $\mathrm{dRe} / \mathrm{dt}=0.06$, six steady pairs of vortices develop at $\mathrm{Re}$ $=340$, whereas when the acceleration is $\mathrm{dRe} / \mathrm{dt}=1.3$, seven steady pairs of vortices appear at $R e=520$ and eight pairs at $R e=730$. It is mentioned that the vortices number is very sensitive to the apex angle [5-8]. More recently, $\mathrm{Xu}$ et al. [11] showed in their numerical simulations that the maximum velocity and the pressure magnitudes decrease with increasing the cone apex angle. $\mathrm{Xu}$ et al. [12] observed that the behavior of the

Corresponding author: yahi.fatma@gmail.com 
conical flow is dominated by a competition between the meridional flow and the radial flow. Li et al. [12] studied the transition to Taylor vortices between rotating conical cylinders in the case of rigid boundaries, where both end plates are stationary.

The main objective of this paper is to highlight the critical thresholds corresponding to the onset of the different instabilities occurring in the flow between two vertical coaxial cones. By means of visualization techniques, we were able to show the different transition modes with their characteristics. Using images processing, we were able to plot the spatio-temporal diagrams corresponding to these instabilities of the laminar-turbulent transition regime. The results are compared to those of Wimmer et al. [1-3].

\section{Experimental setup}

The experimental device consists of two coaxial cones made of insulating and transparent material (Plexiglas) in order to allow a good visualization of flow regime. Both cones have the same apex angle $\Phi=12^{\circ}$ giving a constant annular gap $\delta=\mathrm{d} / \mathrm{R}_{1 \max }$ where $\mathrm{d}=(4.85 \pm 0.2)$ $\mathrm{mm}$. The inner cone can rotate and the outer cone is maintained at rest [14].

Our system is characterized by an outer cone with largest radius $\mathrm{R}_{2 \max }=(45 \pm 0.2) \mathrm{mm}$ and lowest radius $\mathrm{R}_{2 \min }=(12 \pm 0.2) \mathrm{mm}$. The largest radius of the inner cone is $R_{1 \max }=(40.15 \pm 0.2) \mathrm{mm}$, while the lowest radius is $\mathrm{R}_{1 \mathrm{~min}}=(7.15 \pm 0.2) \mathrm{mm}$. The length of the fluid column is fixed at $\mathrm{H}=(155 \pm 0.2) \mathrm{mm}$. The inner cone is driven by a DC motor connected to the rotating axis by a flexible in order to avoid the adverse effects of vibration (Fig. 1).

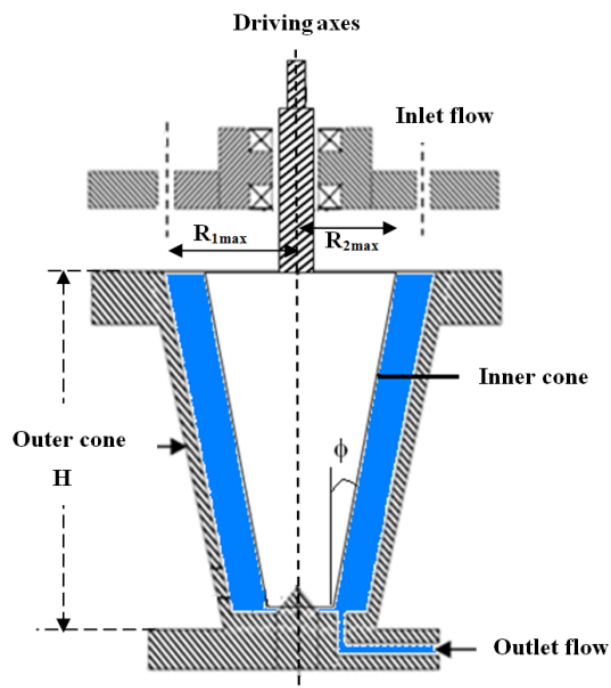

Fig. 1: Conical Taylor-Couette flow system.

The working fluid is a solution of $20 \%$ of Vaseline oil CHALLALA, favoring a better suspension of the particles in the fluid visualization, which is added to $80 \%$ of a petroleum product SIMILI to reduce the viscosity of the oil, with a concentration of $2 \mathrm{~g} / \mathrm{l}$ of aluminum flakes. Such a mixture constitutes a Newtonian fluid characterized by its kinematic viscosity $v=4.810^{-6} \mathrm{~m}^{2} / \mathrm{s}$ and its density $\rho=777.23 \mathrm{~kg} / \mathrm{m}^{3}$ with an accuracy of $1 \%$.

In order to characterize the onset of the hydrodynamic instabilities, it is necessary to introduce dimensionless numbers involving viscous forces, which play a stabilizing role, and centrifugal forces, which have a destabilizing effect. These dimensionless numbers, which serve as control parameters of the flow, are the Reynolds number $\mathrm{Re}$, the Taylor number $\mathrm{Ta}$ and the Froude number Fr defined in Table 1.

Table 1. Control parameters.

\begin{tabular}{|c|c|}
\hline Reynolds number & $\mathrm{Re}=\frac{\mathrm{R}_{1 \mathrm{max}} \Omega \mathrm{d}}{v}$ \\
\hline Taylor number & $\mathrm{Ta}=\operatorname{Re} \delta^{1 / 2}$ \\
\hline Froude number & $\mathrm{Fr}=\frac{\mathrm{R}_{1 \max } \Omega}{\sqrt{\operatorname{gd} \cos \phi}}$ \\
\hline
\end{tabular}

The manifestation of a given wave form or instability was identified with three visualization techniques [14], which are now presented.

\subsection{Reflection of natural light}

This method is based on the light beam reflection on the seeding particles. The light is supplied by an external source located in front of the experimental device. The intensity of the reflected light will depend on the orientation of the aluminium flakes, which are aligned with the local velocity vector. As a consequence, if the velocity vector is axial, the flakes will reflect light strongly and bright zones will appear on the images. On the contrary, if the velocity has a significant radial component, the flakes will be oriented parallel to the light rays and will let the light pass without reflection, giving dark zones on the images. However, as shown by Wimmer [3], two successive vortices have different orientations (Fig. 2), so that only one dark zone out of two is clearly visible on the images. In the interval between these well visible dark zones, two counterrotating vortices are then present.

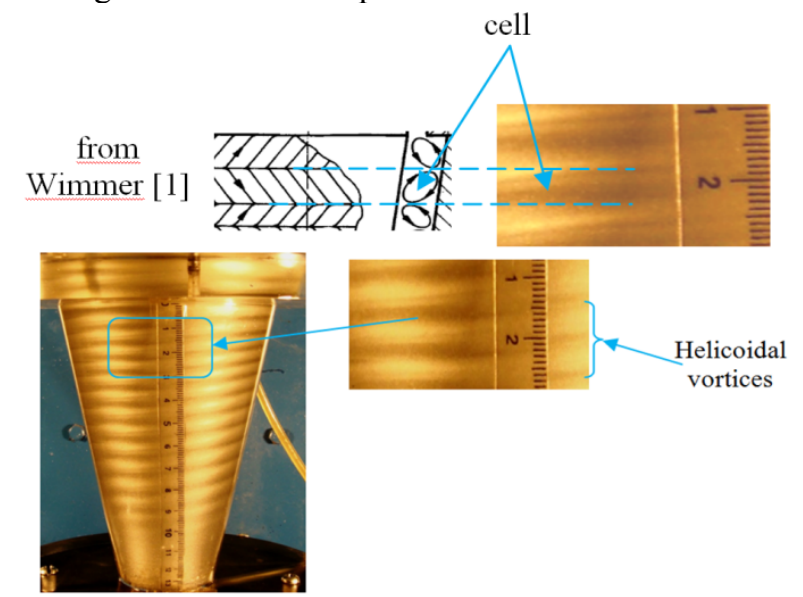

Fig. 2: Natural light reflection $(\delta=0.12, \mathrm{Ta}=79)$. 


\subsection{Transmission of natural light}

This method of visualization is based on the optical transmission of the light source, which is placed behind the experimental device. The light rays pass through the flow and provide in-depth structure of the flow. In contrast with the previous technique, dark zones correspond to axial velocity vectors and bright zones to radial orientations, and all the dark and bright zones are well visible (Fig. 3).

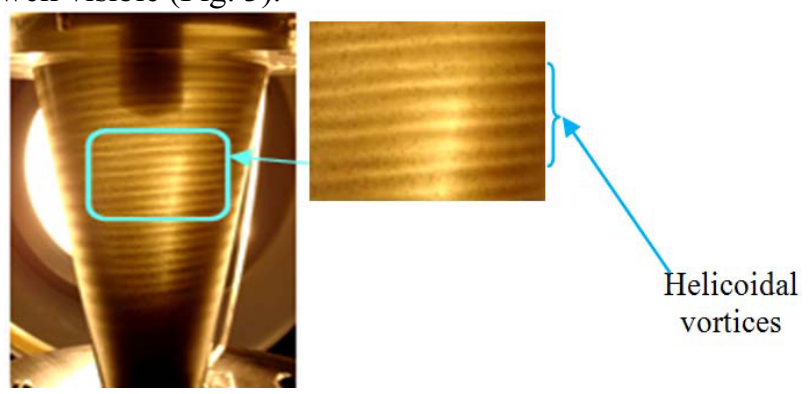

Fig. 3: Natural light transmission $(\delta=0.12, \mathrm{Ta}=72)$.

\subsection{Laser transmission}

Finally, in order to examine the local structure of the flow and better see the vortices, we have used a coherent light (laser $\mathrm{He}, 1 \mathrm{~mW}$ and with wavelength $\lambda=623.8 \mathrm{~nm}$ ), which is expanded as a laser sheet thanks to a cylindrical lens. This laser sheet illuminates an azimuthal plane in order to see the global vortex structure in this plane or to isolate certain vortices. Adequate settings of the optics are necessary in order to obtain good visualizations with a rigorous stigmatism of the images (Fig. 4a-4b).

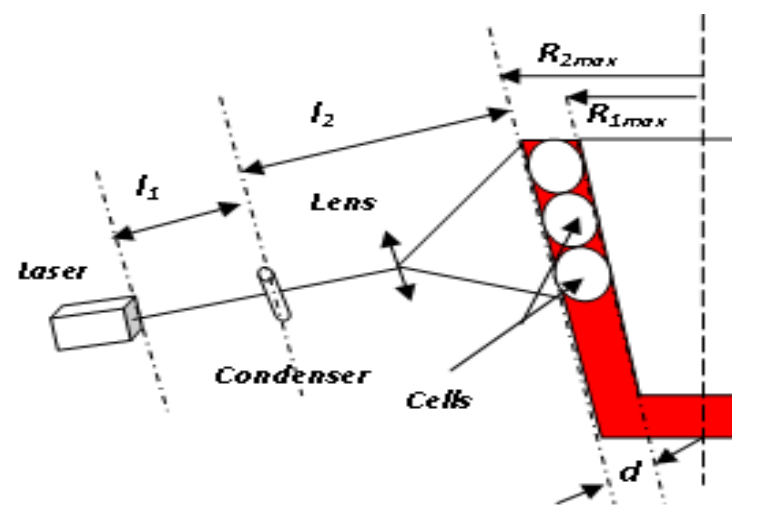

a)

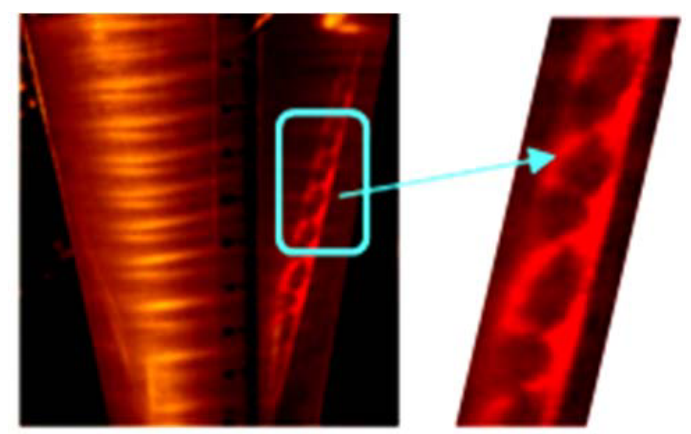

b)

Fig. 4: a) Laser transmission visualization layout; b) Laser transmission visualization $(\delta=0.12, \mathrm{Ta}=88.5)$.

\section{Results and discussions}

For the small gap configuration, $\delta=0.12$, and for different angular velocity, we investigate the basic flow and the laminar-turbulent transition regime for Taylor numbers up to $\mathrm{Ta}=318.1$.

\subsection{Basic flow}

The basic flow, in the absence of any disturbances, is laminar, three-dimensional $\left(\mathrm{V}_{\Theta}, \mathrm{V}_{\mathrm{r}}\right.$ and $\mathrm{V}_{\mathrm{z}}$ velocity components) and axi-symmetric (Fig. 5). The threedimensional character of the flow is the result of the imbalance of the centrifugal forces in the annulus: indeed, the linear variation of the radius versus the conical axial position $\mathrm{z}$ induces a linear variation of these centrifugal forces. The flow observed in any azimuthal plane corresponds to a large loop going up along the inner cone and down along the outer cone and turning at the end plates [1]. These end plates being fixed with no-slip conditions, the centrifugal forces decrease in their vicinity. This effect will promote end recirculations, in the direction of the main loop at the bottom and in the opposite direction at the top. The recirculation at the bottom will only reinforce the main loop. In contrast, the recirculation at the top will progressively generate a vortex in counter-rotation with the main loop. Due to the axi-symmetry of the flow, this vortex (known as Ekman vortex) is in fact a toroidal cell. In the small gap situations considered here, it has not been possible to provide evidence of this Ekman vortex.

A reason could be the difficulty to visualize the upper zone of the annulus, which is behind a large thickness Plexiglas zone (Fig. 1).
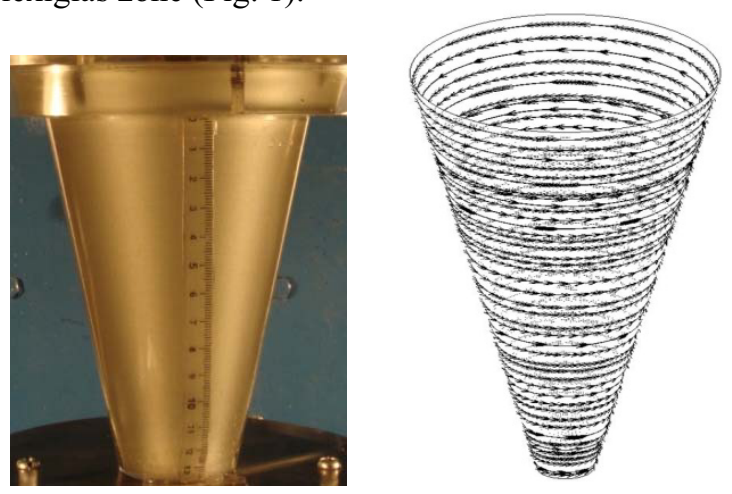

Fig. 5: Basic flow $\left(\delta=0.12, \mathrm{Ta}<\mathrm{Ta}_{\mathrm{c} 1}\right)$.

\subsection{Transition regimes}

By progressively increasing the angular velocity of the inner cone (corresponding to an increase of the Taylor number), we observe the development of disturbances in the upper part of the basic flow for $\mathrm{Ta}>\mathrm{Ta}_{\mathrm{cl}}=42.3$. These disturbances correspond to the well-known Taylor vortices (TV), which generally appear as steady regular closed vortex cells. In our case, the first Taylor cell appears in the vicinity of the upper edge, where the centrifugal forces are greater. This toroidal cell is stationary and its size is of the order of the annular gap. It forms a pair of cells with the rest of the flow, which is unperturbed and remains a large loop (UF) (Fig. 6). Only 
the first Taylor cell will be visible, before the development of the next instability.

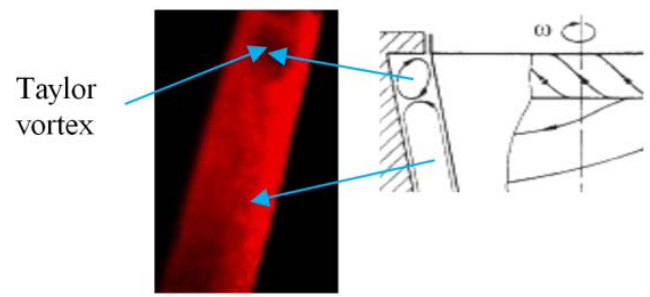

Fig. 6: Laser transmission visualization of the Taylor vortex (TV) $\left(\mathrm{Ta}=\mathrm{Ta}_{\mathrm{c} 1}=42.3\right)$.

By still increasing the inner cone velocity gradually, we observe the appearance of new cells, which propagate downwards as a helical motion (DHM) (Fig. 7). This phenomenon occurs at $\mathrm{Ta}=\mathrm{Ta}_{\mathrm{c}}{ }^{\mathrm{DHM}}=47.2$. The downward movement of the vortices seems to be related to the corresponding decrease of the radius in our geometry, as experiments performed in the up side down configuration have given an upward movement of the vortices. Note that, as for the Taylor vortices, there remain an unperturbed zone (UF), corresponding to a large loop, at the bottom part of the annulus. This unperturbed zone will only disappear for far larger Taylor numbers, as for $\mathrm{Ta}=192$ (see Fig. 9). For Ta above $\mathrm{Ta}_{\mathrm{c}}{ }^{\mathrm{DHM}}$ and before the next instability, three different flow structures can be observed in the annulus: from top to bottom, the steady Taylor vortex (TV), the downward helical vortices (DHM) and the unperturbed flow loop (UF).

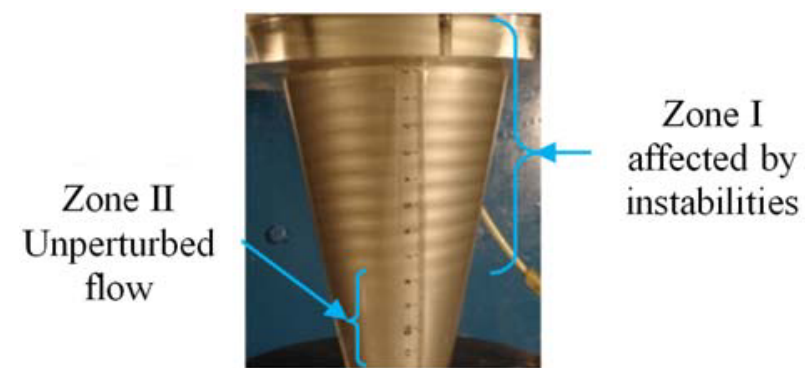

Fig. 7: Visualization of the downward helical motion (DHM) at $\mathrm{Ta}=61.1$.

A wavy mode (WM) corresponding to an azimuthal wave appears in the very upper part of the annulus with a very low amplitude at $\mathrm{Ta}=\mathrm{Ta}_{\mathrm{c} 2}=68.8$. The other parts of the annulus keep the same flow properties as those described above. The wavy mode first affects the Taylor vortex, but for increasing $\mathrm{Ta}$, it will also affect the downward helical motion (see Fig. 9f). Finally, for Ta $=$ $\mathrm{Ta}_{\mathrm{c}}{ }^{\mathrm{UHM}}=72$, we observe the appearance of upward helical motions of cells. At this value of Ta, we observe the co-existence of the Taylor vortex with wavy mode, the downward helical motion, the upward helical motion and the unperturbed flow at the bottom (Fig. 8). At larger $\mathrm{Ta}$, we will note the possible superposition of three flow instabilities in some zones of the annulus, namely, the downward helical motion (DHM), the upward helical motion (UHM) and the wavy mode (WM).

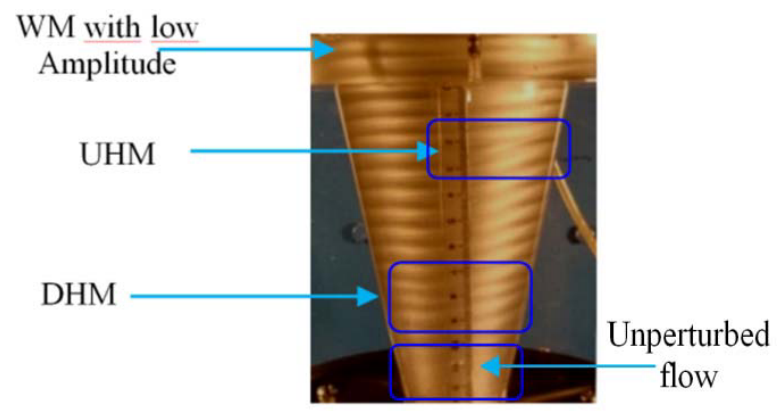

Fig. 8. Co-existence of four flow regimes: wavy mode (WM) affecting the Taylor vortex, the upward helical motion (UHM), the downward helical motion (DHM), and the unperturbed flow (UF) $(\mathrm{Ta}=80.6)$.

By observation of Table 2, we can see that the results obtained with our experimental device for $\delta=0.12$ are in good agreement with those of Wimmer [1] obtained for $\delta$ $=0.1$. Wimmer [1] observed the Taylor vortex, the downward helical motion and the wavy mode for values of Ta close to ours.

Table 2: Comparison of the critical Taylor number values for different gap configurations (completely filled flow system).

\begin{tabular}{|c|c|c|c|c|}
\hline $\begin{array}{c}\text { Critical } \\
\text { Taylor } \\
\text { number }\end{array}$ & $\mathbf{T a}_{\mathbf{c 1}}$ & $\mathbf{T a c}^{\mathbf{D H M}}$ & $\mathbf{T \mathbf { a } _ { \mathbf { c } 2 }}$ & $\mathbf{T a}_{\mathbf{c}}{ }^{\mathbf{U H M}}$ \\
\hline $\begin{array}{c}\text { Flow } \\
\text { regimes }\end{array}$ & $\mathbf{T V}$ & $\mathbf{D H M}$ & $\mathbf{W M}$ & $\mathbf{U H M}$ \\
\hline $\begin{array}{c}\text { Wimmer [1] } \\
\delta=0.1\end{array}$ & 41.6 & 50 & 74.9 & \\
\hline $\begin{array}{c}\text { Present } \\
\text { work } \\
\delta=0.12\end{array}$ & 42.3 & 47.2 & 68.8 & 72 \\
\hline
\end{tabular}

Some flow states showing the instability modes in a larger range of Ta values are presented in Fig. 9. We can note the larger amplitude of the wavy mode for the larger values of Ta.

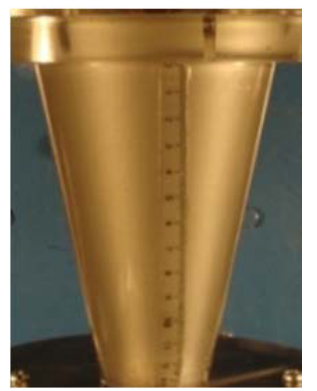

a) $\mathrm{Ta}<42.3$ basic laminar flow

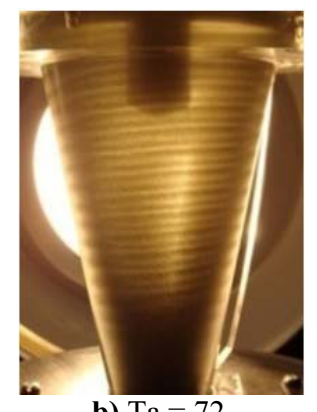

b) $\mathrm{Ta}=72$ DHM 


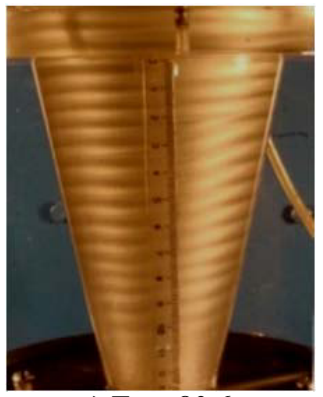

c) $\mathrm{Ta}=80.6$ DHM+WM+UHM

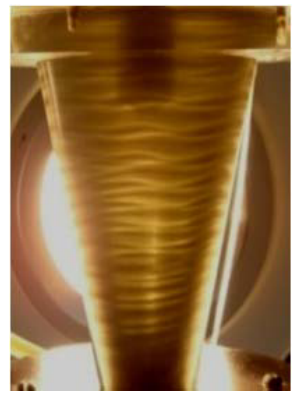

e) $\mathrm{Ta}=130.5$

$\mathbf{D H M}+\mathbf{W M}+\mathbf{U H M}$

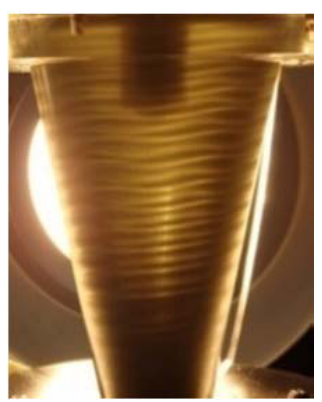

d) $\mathrm{Ta}=89.6$ DHM+WM+UHM

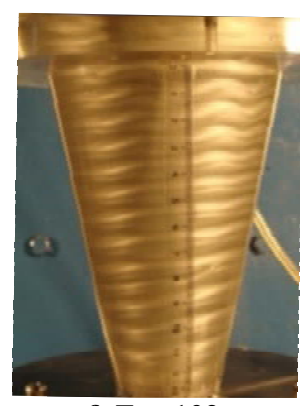

DHM+WM+UHM f) $\mathrm{Ta}=192$

Fig. 9: Flow state visualizations for different Ta showing the different flow regimes: a), c) and f) Natural light reflection; b), d) and e) Natural light transmission $(\delta=0.12)$.

Finally, we can remark that the helical structure appears on previously obtained axi-symmetric regimes (i.e. the basic flow and Taylor vortex regimes) and breaks this axi-symmetry. This indicates that the corresponding instability must be an azimuthal mode with a non-zero azimuthal wavenumber $\mathrm{m}$. In fact, by accurately analyzing the images of downward helical motion (as in Fig. 10), we have found that a pair of counter-rotating vortices is shifted down by a height equal to its wavelength after a -azimuthal rotation (which is what we can see on the front view of the cone on the images). By extrapolation (to take into account the shift occurring on the back face of the cone), we deduce that the pair of vortices is shifted by two wavelengths for a turn around the cone, which indicates that the helical instability corresponds to an azimuthal mode $m=2$. Comparisons, however, were not possible as we did not find any indication of azimuthal wavenumbers in the previous publications on rotating cones. Numerical stability analyses could be performed to clarify this point.

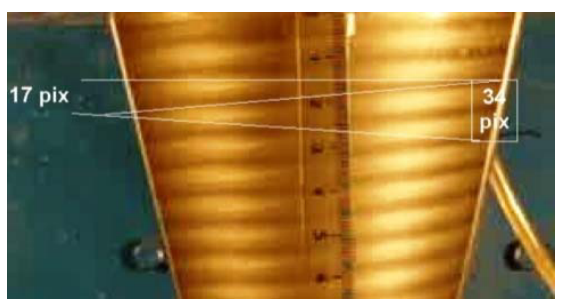

Fig. 10: Analysis of the downward helical motion to determine the azimuthal wavenumber of the governing instability.

\subsection{Evolution of cells number and wavelength for different flow regimes}

The analysis of the results allowed us to determine the evolution of the cells number $\mathrm{n}$ according to the Taylor number Ta and the flow regime. The curve showing $n$ versus $\mathrm{Ta}$ is shown in Fig. 11. The vortices number $\mathrm{n}$ starts at one (corresponding to the Taylor vortex) in the vicinity of the first instability at $\mathrm{Ta}=42.3$. It then increases quite quickly to reach its maximum value $n=36$ at $\mathrm{Ta}=78.3$. This steep increase corresponds to the progressive downward extension of the helical structure as $\mathrm{Ta}$ is increased. The vortices number $\mathrm{n}$ keeps the same value $n=36$ on a large Ta gap, up to $T a=161$, indicating that, for all these states, the helical structure has reached the bottom of the cone. At $\mathrm{Ta}=192$, m slightly decreases to $\mathrm{m}=34$, with the disappearance of a pair of counterrotating cells. Something similar is observed at $\mathrm{Ta}=276.7$, and the new structure with $\mathrm{m}=32$ is obtained up to $\mathrm{Ta}=318.1$, the limit $\mathrm{Ta}$ value in our study.

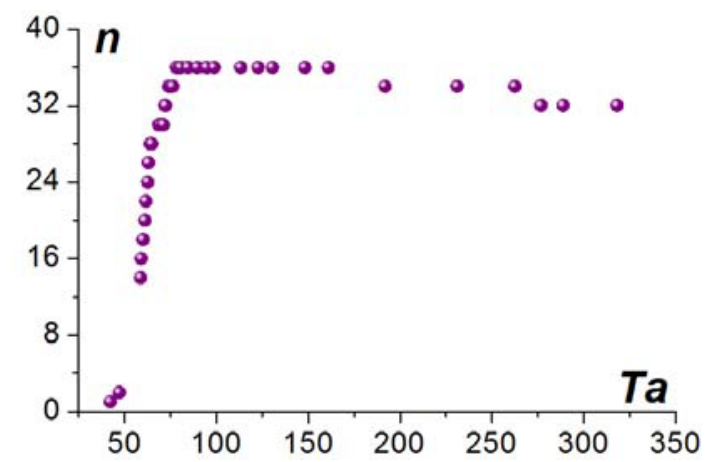

Fig. 11: Variation of the cell number $n$ versus the Taylor number Ta.

By using image processing with Matlab, we were able to extract spatio-temporal diagrams from the videos available for some of the flow states (sequences of 100 to 200 images are chosen, covering time intervals of 9 to 13 seconds). For each image of the videos, we have registered the grey levels along a given generatrix of the cone ( 280 pixels along a generatrix). If the generatrix determined by the vertical plane perpendicular to the image is taken as a reference (this generatrix corresponds to the vertical line in the middle of the image), the generatrix chosen for the spatio-temporal diagrams presented in Fig. 12 is the generatrix at - /6 (on the left part of the image) and for the diagram in Fig. 13, it is the generatrix at $/ 8$ (on the right part of the image). These diagrams are presented as color levels in a domain with the time (in seconds) in abscissa and the position along the generatrix (in $\mathrm{mm}$ and with the origin at the bottom of the cone) in ordinate. They will allow us to determine the wavelength and the drift velocity of the vortices. 

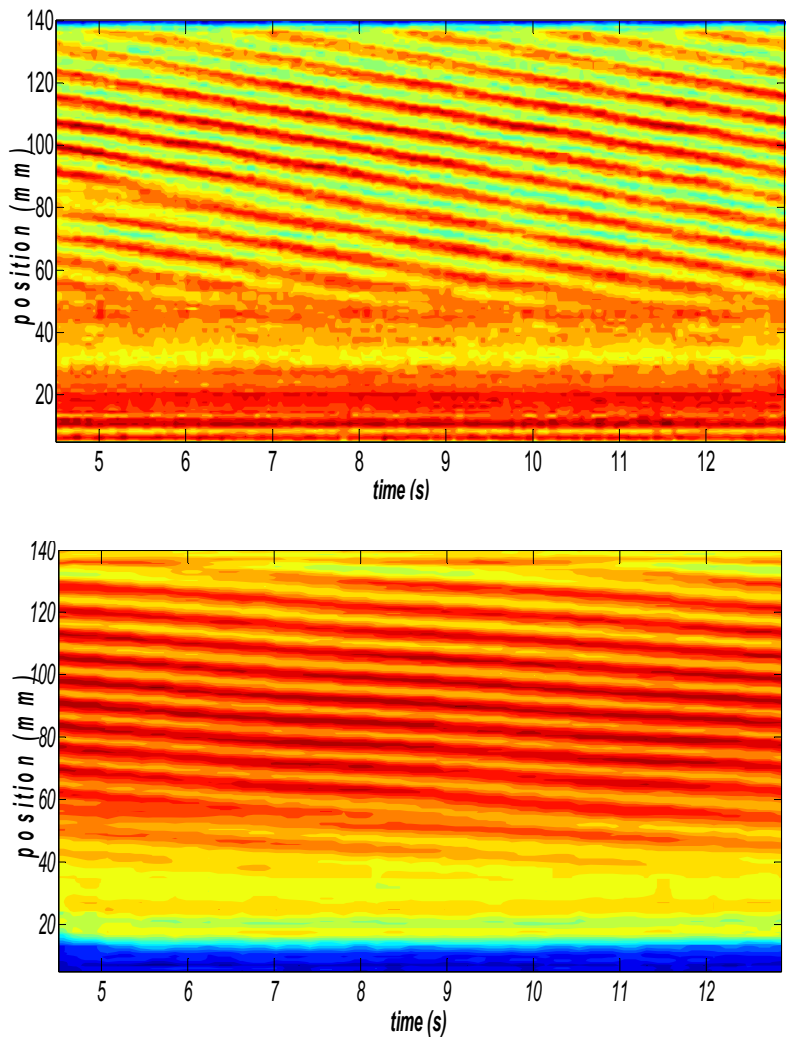

Fig. 12: Spatio-temporal diagrams showing the downward helical motion for $\mathrm{Ta}=64$ (first view) and 74 (second view).

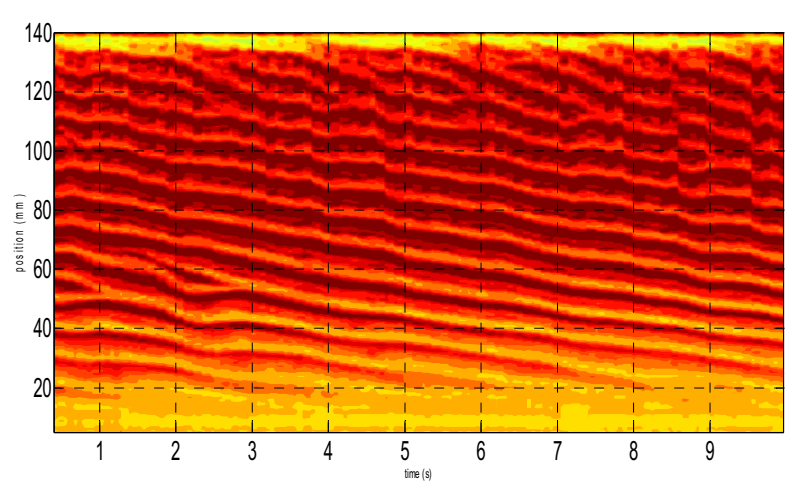

Fig. 12: Spatio-temporal diagram for $\mathrm{Ta}=89.6$.

In figure 12, the downward helical motion is clearly put in evidence for $\mathrm{Ta}=64$ and 74 . In both cases, there also remains an unperturbed flow zone at the bottom of the cone. Comparisons between the two cases (which are presented in domains with the same scale) show that the drift velocity decreases when Ta is changed from 64 to 74. At $\mathrm{Ta}=74$, a case above the different instability thresholds, the wavy mode is hardly visible (except, may be, close to the top) and the upward helical motion not clearly present.

In contrast, for $\mathrm{Ta}=89.6$, we clearly observe the coexistence of the downward helical motion, the wavy mode and the upward helical motion. The wavy mode affects almost all the cells: it has, however, a large amplitude for the cells in the upper part of the cone and this amplitude decreases as the cells reach lower zones.
The upward helical motion can be seen through the sudden disappearance of a pair of counter-rotating vortices, which appears in the diagram as a dislocation. From the time interval between two successive dislocations $(T \approx 1.64 s)$ and the fact that it generally affects the next but one pair of cells (two wavelengths apart, i.e. $\mathrm{L}=2 \lambda \approx 3.87 \mathrm{~mm}$ ), we can estimate a propagation velocity for this upward helical motion at $2.36 \mathrm{~mm} / \mathrm{s}$.

The analysis of these spatio-temporal diagrams allowed us to determine the wavenumber $\mathrm{k}$ of the cell structure by Fourier transform and to deduce the wavelength $\lambda$. For example, for $\mathrm{Ta}=61$ (a case with a downward helical motion of the cells), the wavenumber is $\mathrm{k}=0.1067$ giving a wavelength $\lambda=9.366 \mathrm{~mm}$, which is close to $2 \mathrm{~d}=9.7 \mathrm{~mm}$. The results obtained for $\lambda^{*}=$ $\lambda /(2 d)$ in a Ta range from 60 to 90 are given in Fig. 14 . The increase of the Taylor number induces a decrease of the wavelength $\lambda^{*}$, which becomes almost constant for Ta above approximately 70 at a value close to 0.77 $(\lambda \approx 7.5 \mathrm{~mm})$.

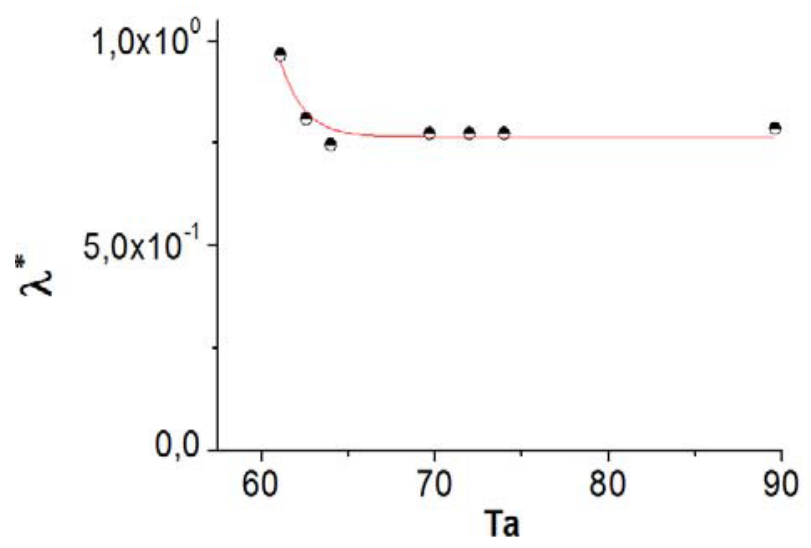

Fig. 14: Evolution of the dimensionless wavelength $\lambda$ versus the Taylor number.

The dimensionless drift velocity is given by $V_{d}{ }^{*}=$ $V_{d} /\left(R_{1 \max } \Omega\right)$, where $V_{d}=\lambda / T, \lambda$ is the wavelength and $\mathrm{T}$ is the period of the flow signal. The variation of this drift velocity with the Taylor number is presented in Fig. 15. $V_{d}$ is found to decrease when the Taylor number is increased. For Ta in the range 60 to 80 , this decrease is close to a linear decrease, in good agreement with what was observed by Wimmer [1]. 


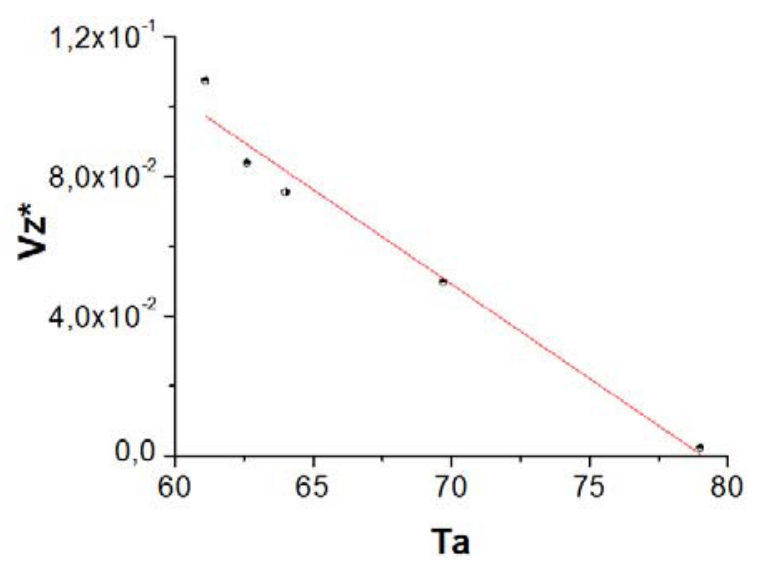

Fig. 15: Evolution of the dimensionless drift velocity versus the Taylor number.

\section{Conclusion}

The study of the laminar-turbulent transition in the conical Taylor-Couette flow system was motivated by the interest for different industrial applications and by the hope to discover a specific laminar-turbulent transition. The present experiments, based on optical visualization techniques, indicate that the chaotic regime occurs after a small number of transitions to distinct modes: the Taylor vortex mode, the downward helical motion, the wavy mode and the upward helical motion. The results are in good agreement with those obtained by Wimmer [1-3] in the case $\delta=0.11$. Spatio-temporal diagrams were also obtained by image processing. The spectral analysis performed on such diagrams enabled us to determine the wavelength and the corresponding drift velocity of the downward moving cells in the interval 60 $<\mathrm{Ta}<90$. It was noted that, when $\mathrm{Ta}$ is increased, the wavelength decreases towards a constant value, whereas the drift velocity follows a linear decrease.

F.H. acknowledges the PROFAS B+ fellowship allowing this international collaboration work.

\section{References}

1. M. Wimmer, J. Fluid Mech. 292, 205-227 (1995).

2. M. Wimmer, J. Zierep, Acta Mech. 140, 17-30 (2000).

3. M. Wimmer, Prog. Aerospace Sci. 25, 43-103 (1988).

4. M.N. Noui-Mehidi, M. Wimmer, Acta Mech. 135, 13-25 (1999).

5. M.N. Noui-Mehidi, N. Ohmura, K. Kataoka, Int. J. Fluid Dyn. 5, Article 1 (2001).

6. M.N. Noui-Mehidi, N. Ohmura, K. Nishiyama, T. Takigawa, Commun. Nonlinear Sci. Num. Sim. 14, 613-621 (2009).

7. M.N. Noui-Mehidi, N. Ohmura, K. Kataoka, $12^{\text {th }}$ Int. Couette-Taylor Workshop, September 6-8 (2001).

8. M.N. Noui-Mehidi, N. Ohmura, K. Kataoka, J. Fluids Struct. 16, 247-262 (2002).
9. M.N. Noui-Mehidi, N. Ohmura, K. Kataoka, J. Fluids Struct. 20, 331-334 (2005).

10. N. Ohmura, M.N. Noui-Mehidi, K. Sasaki, K. Kitajima, K. Kataoka, J. Chem. Eng. Japan 37, 546550 (2004).

11. X.F. Xu, L.X. Xu, Commun. Nonlinear Sci. Num. Sim. 14, 2670-2676 (2009).

12. X.F. Xu, P. Wen, L.X. Xu, D.P. Cao, Commun. Nonlinear Sci. Num. Sim. 15, 1228-1239 (2010).

13. Q.S. Li, P. Wen, L.X. Xu, J. Hydro. 22, 241-245 (2010).

14. F. Yahi, Y. Hamnoune, A. Bouabdallah, S. Lecheheb, F. Mokhtari, J. Applied Fluid Mech. 9, $\mathrm{N}^{\circ} 6$ (to be published, 2016). 